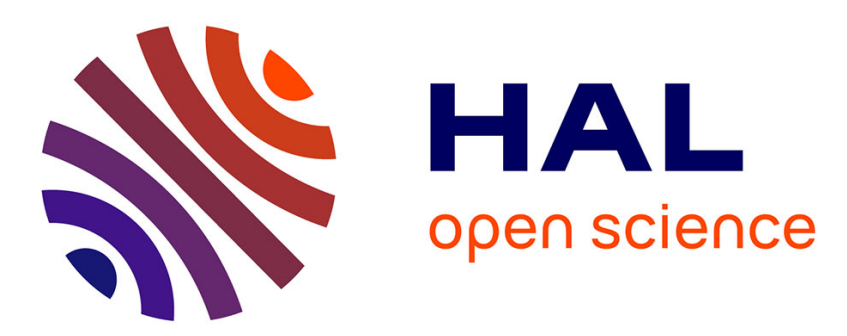

\title{
Minimum Implicational Basis for -Semidistributive Lattices
}

\author{
Philippe Janssen, Lhouari Nourine
}

\section{To cite this version:}

Philippe Janssen, Lhouari Nourine. Minimum Implicational Basis for -Semidistributive Lattices. Information Processing Letters, 2006, 99 (5), pp.199-202. 10.1016/j.ipl.2006.04.004 • lirmm-00101743

\section{HAL Id: lirmm-00101743 https://hal-lirmm.ccsd.cnrs.fr/lirmm-00101743}

Submitted on 28 Sep 2006

HAL is a multi-disciplinary open access archive for the deposit and dissemination of scientific research documents, whether they are published or not. The documents may come from teaching and research institutions in France or abroad, or from public or private research centers.
L'archive ouverte pluridisciplinaire HAL, est destinée au dépôt et à la diffusion de documents scientifiques de niveau recherche, publiés ou non, émanant des établissements d'enseignement et de recherche français ou étrangers, des laboratoires publics ou privés. 


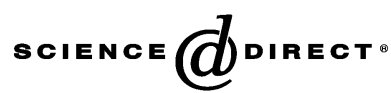

Information Processing Letters $\bullet \bullet \bullet(\bullet \bullet \bullet \bullet) \bullet \bullet \bullet-\bullet \bullet \bullet$
Information Processing ${ }^{53}$ Letters

www.elsevier.com/locate/ipl

\title{
Minimum implicational basis for $\wedge$-semidistributive lattices
}

\author{
Philippe Janssen ${ }^{\mathrm{a}}$, Lhouari Nourine ${ }^{\mathrm{b}, *}$ \\ a LIRMM, Université Montpellier II, 161, rue Ada, F34392 Montpellier cedex 5, France \\ b LIMOS, Université Blaise Pascal, Campus des cézeaux, F63173 Aubiere, France
}

Received 13 January 2006; received in revised form 21 February 2006; accepted 21 April 2006

Communicated by L. Boasson

\begin{abstract}
For a $\wedge$-semidistributive lattice $L$, we study some particular implicational systems and show that the cardinality of a minimum implicational basis is polynomial in the size of join-irreducible elements of the lattice $L$. We also provide a polynomial time algorithm to compute a minimum implicational basis for $L$.

(c) 2006 Published by Elsevier B.V.

Keywords: Algorithms; Lattice; Closure system; Minimum implicational basis
\end{abstract}

\section{Introduction}

This paper deals with the computation of a minimum implicational basis for a closure system. Computing a minimum implicational basis for a lattice given by its poset of irreducible elements is an important problem, which has applications to many areas of computer science, in particular to databases and $\mathrm{AI}[1,4,6,7,10]$. For a survey on this problem and related areas, see [3].

The complexity of this problem remains open for general lattices. Recent progress on the status of this problem, and in particular solvability by limited nondeterminism [5], suggests however that this problem is more likely to be expected tractable than intractable [4].

It has been already shown that this problem is tractable for the two classes of locally distributive lattice [2] and of modular lattices [14]. In this paper we

\footnotetext{
* Corresponding author.

E-mail addresses: pja@lirmm.fr (P. Janssen), nourine@ isima.fr
} (L. Nourine). show by using a dependence relation in [11] that the class of $\wedge$-semidistributive lattices is another tractable case.

Consider a finite set $U$. A subset $\mathcal{C} \subseteq 2^{U}$ is said to be a closure system if $\mathcal{C}$ is closed under set-intersection and containing the set $U$. An implication on $U$ is an ordered pair $(A, B)$ of subsets of $U$, denoted by $A \rightarrow B$. The set $A$ is called the premise and the set $B$ the conclusion of the implication $A \rightarrow B$. Let $\Sigma$ be a set of implications on $U$. A subset $D \subseteq U$ is $\Sigma$-closed if for each implication $A \rightarrow B$ in $\Sigma, A \subseteq D$ implies $B \subseteq D$. The set of $\Sigma$-closed subsets of $U$, denoted by $\mathcal{C}(\Sigma)$, is a closure system on $U$. Conversely, given a closure system $\mathcal{C}$ on $U$, a family $\Sigma$ of implications on $U$ is said an implicational basis for $\mathcal{C}$ if $\mathcal{C}=\mathcal{C}(\Sigma)$. An implicational basis is said minimum if it has a minimum number of implications.

In this paper, we study the latticial version of this problem. We view a lattice $L$ as the closure system $\mathcal{C}_{L}$ on the set $J(L)$ of its join-irreducible elements. More precisely, put $J(a)=\{j \in J(L): j \leqslant a\}$ for $a \in L$.

0020-0190/\$ - see front matter (C) 2006 Published by Elsevier B.V. 


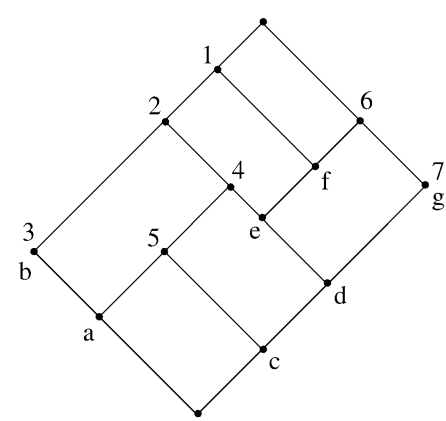

(b)

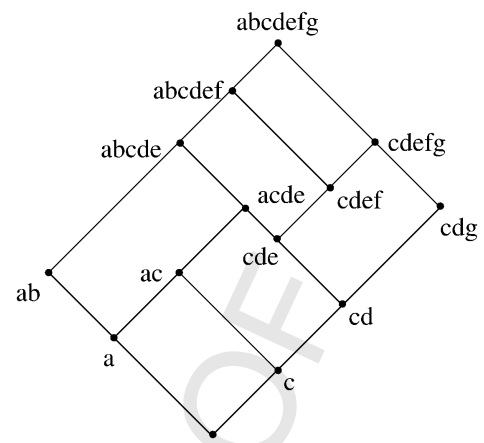

(b)

Fig. 1. (a) A lattice $L$ where join-irreducible (resp. meet-irreducible) elements are labeled by letters (resp. numbers); (b) The closure system $c l$ associated to $L$.

Then $\mathcal{C}_{L}=\{J(a): a \in L\}$ is a closure system on $J(L)$ which, as a lattice ordered by inclusion, is isomorphic to $L$.

Fig. 1 gives an example of the closure system $\mathcal{C}_{L}$ associated to a lattice $L$.

The closure system $\mathcal{C}_{L}$ can be defined by the set of its meet-irreducible elements $\mathcal{M}\left(\mathcal{C}_{L}\right)=\{J(m): m \in$ $M(L)\}$, where $M(L)$ denotes the set of meet-irreducible elements of $L$. Each element of $\mathcal{C}_{L}$ can be obtained as intersection of some elements of $\mathcal{M}\left(\mathcal{C}_{L}\right)$.

The problem we study is:

Problem: Minimum implicational basis

Instance: The set of meet-irreducible elements $\mathcal{M}\left(\mathcal{C}_{L}\right)$ of the closure system $\mathcal{C}_{L}$.

Question: Find a minimum basis $\Sigma$ for $\mathcal{C}_{L}$.

This problem remains open for general lattices. Duquenne [2] has given a latticial version of this problem and shown that it is polynomial for upper locally distributive lattices or antimatroid. Recently, Wild [14] has proposed a polynomial time algorithm to compute an optimal ${ }^{1}$ implicational basis for modular lattices. In the following, we study the case of $\wedge$-semidistributive lattices. For such lattices we show that the number of implications of a minimum implicational basis is at most $|J(L)|^{2}$ and give a polynomial time algorithm to compute such a basis.

\section{Some properties of $\wedge$-semidistributive lattices}

Let $L$ be a finite lattice. We note $\vee$ the join operation, $\wedge$ the meet operation and $\prec$ the cover relation of $L$. If $j$ is a join-irreducible element of $L$, we use $j_{*}$ to denote

1 An implication is known as optimal if the sum of the cardinality of the premises and the conclusions of all the implications is minimal. the unique element covered by $j$. Dually, we use $m^{*}$ to denote the unique element covering a meet-irreducible element $m$.

We will use the arrow relations introduced by Wille [15]: for $x, y \in L, x \downarrow y$ means that $x$ is a minimal element of $\{z \in L: z \nless x\}, x \uparrow y$ means that $y$ is a maximal element of $\{z \in L: z \not y\}$ and $x \uparrow y$ means that $x \uparrow y$ and $x \downarrow y$. Recall that $\uparrow, \downarrow, \uparrow$ are relations defined on $J(L) \times M(L)$, where $J(L)$ is the set of join-irreducible elements and $M(L)$ the set of meet-irreducible elements of $L$.

In the following, we deal essentially with $\wedge$-semidistributive lattices. Let us recall that a lattice $L$ is said $\wedge$-semidistributive if for all elements $x, y, z \in L, x \wedge$ $y=x \wedge z$ implies $x \wedge y=x \wedge(y \vee z)$. A $\wedge$-semidistributive lattice is said semidistributive if for all elements $x, y, z, x \vee y=x \vee z$ implies $x \vee y=x \vee(y \wedge z)$. The following characterization of these lattices are well known (see, for example, [6]):

Property 1. A finite lattice $L$ is $\wedge$-semidistributive if and only if for any $j \in J(L)$ there exists a unique $m \in$ $M(L)$ such that $j \uparrow m$.

For any $\wedge$-semidistributive lattice $L$ and $j \in J(L)$, we denote by $m(j)$ the unique element $m \in M(L)$ such that $j \uparrow m$.

We define the mapping $\gamma: J(L) \rightarrow 2^{M(L)}$ by $\gamma(j)=$ $\{m \in M(L): j \downarrow m\}$. This mapping was introduced in [12] to define colored posets, which provides a new representation for lattices, and specially for upper locally distributive lattices. Fig. 2 shows the $\gamma$ mapping of the lattice of Fig. 1. Note that this lattice is semidistributive.

We consider one of the standard dependence relations defined on join-irreducible elements (assuming 


\begin{tabular}{|c|c|c|c|c|c|c|}
\hline & 12 & 3 & 4 & 5 & & $\gamma$ \\
\hline$a$ & & & & & & $\{6,7\}$ \\
\hline$b$ & & & & $\downarrow$ & & $\{4,5\}$ \\
\hline$c$ & & $\uparrow$ & & & & $\{3\}$ \\
\hline$d$ & & $\uparrow$ & & $\uparrow$ & & $\{5\}$ \\
\hline$e$ & & $\uparrow$ & & $\uparrow$ & & $\{7\}$ \\
\hline$f$ & $\downarrow$ & & $\downarrow$ & & & $\{2,4\}$ \\
\hline$g$ & $\uparrow \downarrow$ & & $\downarrow$ & & & $\{1,2,4\}$ \\
\hline
\end{tabular}

Fig. 2. The arrow relations and mapping $\gamma$ of the lattice in Fig. 1.
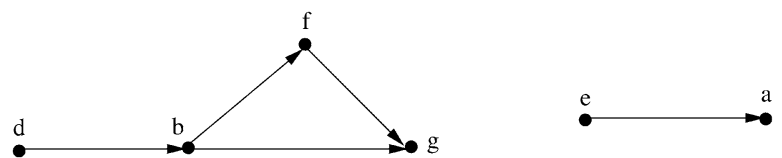

Fig. 3. The graph of the $B$ relation for the lattice in Fig. 1 .

that the lattice $L$ is $\wedge$-semidistributive) as follows (see, for example, $[8,11])$ :

Let $j, j^{\prime} \in J(L)$.

Then $j B j^{\prime} \quad$ iff $\quad j \neq j^{\prime}, j^{\prime} \nless m(j), j_{*}^{\prime} \leqslant m(j)$.

For an illustration of that definition, see Fig. 3.

There are relationships between the existence of cycles in the graph of the relation $B$ and some classes of lattices. For example, Nation has shown that a $\wedge$-semidistributive lattice is semidistributive if and only if it contains no $B$-cycle of length 2 [11].

The following lemma gives a rewriting of the definition of the relation $B$ using the mapping $\gamma$.

Lemma 1. Let $L$ be a $\wedge$-semidistributive lattice, $j, j^{\prime} \in$ $J(L)$.

$j B j^{\prime} \quad$ iff $\quad j \neq j^{\prime}$ and $m(j) \in \gamma\left(j^{\prime}\right)$.

\section{Minimum implicational basis for $\wedge$-semidistributive lattices}

In this section, we give a polynomial time algorithm to compute a minimum implicational basis for a $\wedge$-semidistributive lattice.

We start with two technical lemmas on closed sets of a closure system $\mathcal{C}_{L}$. The first one is obvious since the elements of $\mathcal{C}_{L}$ are order ideals of the induced poset by $J(L)$.

Lemma 2. Let $j, j^{\prime} \in J(L)$ such that $j<j^{\prime}$ and $X \in$ $\mathcal{C}_{L}$. Then $j^{\prime} \in X$ implies $j \in X$.

Consider now a $\wedge$-semidistributive lattice $L$ and $j, j^{\prime} \in J(L)$ such that $j B j^{\prime}$. We denote by $P_{j j^{\prime}}$ the set $J\left(j_{*}\right) \cup J\left(j^{\prime}\right)$.
Lemma 3. Let $L$ be a $\wedge$-semidistributive lattice and $j, j^{\prime} \in J(L)$ such that $j B j^{\prime}$ and $X \in \mathcal{C}_{L}$. Then $P_{j j^{\prime}} \subseteq X$ implies $j \in X$.

Proof. Let $x \in L$ such that $X=J(x)$ and $P_{j j^{\prime}} \subseteq X$. Since $J\left(j_{*}\right) \subset X$ this implies that $j_{*} \vee j^{\prime} \leqslant x$, and then it suffices to prove that $j \leqslant j_{*} \vee j^{\prime}$.

Suppose that $j \nless j_{*} \vee j^{\prime}$ and let $m^{\prime} \in M(L)$ be a maximal element of $\left\{z \in L \mid z \not j\right.$ and $\left.z \geqslant j_{*} \vee j^{\prime}\right\}$. By definition of $m^{\prime}$, we have $j \uparrow m^{\prime}$. Moreover $j \downarrow m^{\prime}$ since $j_{*} \leqslant m^{\prime}$. Thus $j \uparrow m^{\prime}$.

Consider now the meet-irreducible $m(j)$ associated with $j$. Then $j^{\prime} \nless m(j)$ since $j B j^{\prime}$. Thus since $j^{\prime} \leqslant m^{\prime}, m^{\prime}$ and $m(j)$ are two distinct elements such that $j \uparrow m^{\prime}$ and $j \uparrow m(j)$. This contradicts the fact that $L$ is $\wedge$-semidistributive.

We can now define a particular set of implications associated to a $\wedge$-semidistributive lattice $L$. Let $\Sigma_{1}=$ $\{j \rightarrow J(j)\}, \Sigma_{2}=\left\{P_{j j^{\prime}} \rightarrow j \mid j^{\prime} \in J(L)\right.$ and $\left.j B j^{\prime}\right\}$ and $\Sigma=\Sigma_{1} \cup \Sigma_{2}$.

For example, the sets of implications $\Sigma_{1}$ and $\Sigma_{2}$ for the lattice in Fig. 1 are $\Sigma_{1}=\{b \rightarrow a b, d \rightarrow c d, e \rightarrow$ $c d e, f \rightarrow$ cdef, $g \rightarrow c d g\}$ and $\Sigma_{2}=\{a c d \rightarrow e, a b c \rightarrow$ $d$, acdef $\rightarrow b$, acd $\rightarrow b, c d e g \rightarrow f\}$.

The following theorem shows that $\Sigma$ is an implicational basis for $\mathcal{C}_{L}$.

Theorem 1. Let $L$ be $a \wedge$-semidistributive lattice. Then the set of implications $\Sigma$ is an implicational basis for $\mathcal{C}_{L}$.

Proof. We need to show that $\mathcal{C}_{\Sigma}=\mathcal{C}_{L}$.

Let $X \in \mathcal{C}_{L}$. By Lemma $2, X$ is $\Sigma_{1}$-closed. By Lemma $3, X$ is $\Sigma_{2}$-closed. Then $X$ is $\Sigma$-closed and $\mathcal{C}_{L} \subseteq \mathcal{C}_{\Sigma}$.

Now let us show that $\mathcal{C}_{\Sigma} \subseteq \mathcal{C}_{L}$. Let $X \in \mathcal{C}_{\Sigma}$. Let $x_{0}=\bigvee X$, i.e., the least closed set containing $X$. Clearly $X$ is an ideal since it is $\Sigma_{1}$-closed. Suppose that $X \notin \mathcal{C}_{L}$ and let $j$ be a minimal element of $J\left(x_{0}\right) \backslash X$. Since $j \leqslant$ $x_{0}$, we have $x_{0} \nless m(j)$. Moreover $X \nsubseteq J(m(j))$, otherwise one would have $\bigvee X \leqslant m(j)$ and then $\bigvee X \neq x_{0}$ Thus there exists an element $j^{\prime} \in X$ such that $m(j) \in$ $\gamma\left(j^{\prime}\right)$ and therefore $P_{j j^{\prime}} \rightarrow j \in \Sigma$ with $P_{j j^{\prime}} \subseteq X$ and $j \notin X$. Then $X$ is not $\Sigma$-closed, which concludes the proof.

Corollary 1. Let $L$ be a $\wedge$-semidistributive lattice. Then there exists an implicational basis for $\mathcal{C}_{L}$ with at most $|B|+|J(L)|$ implications, where $|B|$ is the number of arcs in the relation $B$. 
Data: Let $L$ be a $\wedge$-semidistributive lattice and $\mathcal{M}\left(C_{L}\right)$ the set of meet-irreducible elements of $\mathcal{C}_{L}$.

Result: A minimum basis $\Sigma$ of the closure system $C_{L}$. begin

$$
\begin{aligned}
& \begin{array}{l}
\Sigma=\emptyset ; \\
\text { for } j \in J(L) \text { do }
\end{array} \\
& \mid \begin{array}{l}
\Sigma=\Sigma \cup\{j \rightarrow \varphi(j)\} ; \\
\text { for } j^{\prime} \in J(L) \text { do }
\end{array} \\
& \mid \begin{array}{l}
P=(\varphi(j)) \backslash\{j\} \cup \varphi\left(j^{\prime}\right) ; \\
\Sigma=\Sigma \cup\{P \rightarrow(P)\} ;
\end{array} \\
& \Sigma=\text { a nonredundant cover of } \Sigma ;
\end{aligned}
$$$$
\text { end }
$$

Algorithm 1. Minimum-Basis $\left(\mathcal{M}\left(C_{L}\right)\right)$.

Clearly the set $\Sigma$ of implications obtained as above is in general not minimum. For instance, for the set $\Sigma$ associated to the lattice in Fig. 1, the implication $a c d g \rightarrow b$ is redundant ${ }^{2}$ and can be removed from $\Sigma$ without changing $\mathcal{C}(\Sigma)$.

In the following we give a polynomial time algorithm to compute a minimum basis for a $\wedge$-semidistributive lattice.

\subsection{Algorithm}

This is based on Theorem 1 and the algorithm in [13]. Indeed, the algorithm in [13] computes a minimum basis (called there a minimum cover) from any given basis in polynomial time.

Let $\mathcal{M}\left(\mathcal{C}_{L}\right)$ be the set of meet-irreducible elements. Consider the closure operator $\varphi: 2^{J} \rightarrow 2^{J}$, with for $X \subseteq$ $J, \varphi(X)=\bigcap\left\{M \in \mathcal{M}\left(\mathcal{C}_{L}\right) \mid X \subseteq M\right\}$. The images of the mapping $\varphi$ are said closed sets, and they correspond to the elements of the closure system $\mathcal{C}_{L}$.

Remark 1. We replaced $P \rightarrow j$ by $P \rightarrow \varphi(P)$ to guarantee the minimality after the calculation of a nonredundant cover of $\Sigma$.

Remark 2. Let us note that Algorithm 1 does not compute the same $\Sigma$ as that of Theorem 1. This to avoid the computation of the relation $B$. But like the whole of the implications calculated by Algorithm 1 contains all implications of Theorem 1 (relative with the preceding remark), this guaranteed to us to have a cover of $\mathcal{C}_{L}$.

Theorem 2. Let $L$ be a $\wedge$-semidistributive lattice. Then Algorithm 1 computes a minimum implicational basis $\Sigma$

2 An implication $A \rightarrow B$ in $\Sigma$ is said redundant in $\Sigma$ if it can be derived using Armstrong rules from $\Sigma \backslash\{A \rightarrow B\}$. of $\mathcal{C}_{L}$ in $\mathrm{O}\left(|J|^{5}+|J|^{3}\left|\mathcal{M}\left(\mathcal{C}_{L}\right)\right|\right)$ time complexity. Moreover, the size of $\Sigma$ is at most $|J(L)|^{2}$ implications.

Proof. Theorem 1 guarantees that $\Sigma$ is a basis for the closure system $\mathcal{C}_{L}$. Since the conclusions of all implications are closed by the mapping $\varphi$, the result in [13] guarantees that a not redundant basis is minimum.

Computing the closure of a set $X \subseteq J(L)$ by $\varphi$ can be done in $\mathrm{O}\left(\left|J(L) \| \mathcal{M}\left(\mathcal{C}_{L}\right)\right|\right)$ time complexity. Thus the total time complexity for computing a basis is in $\mathrm{O}\left(|J(L)|^{3}\left|\mathcal{M}\left(\mathcal{C}_{L}\right)\right|\right)$. Now computing a not redundant basis can be done in $\mathrm{O}\left(|J(L)||\Sigma|^{2}\right)$. Since $\Sigma$ has at most $|J(L)|^{2}$ implications, we conclude that the time complexity of Algorithm 1 is in $\mathrm{O}\left(|J(L)|^{5}+\right.$ $\left.|J(L)|^{3}\left|\mathcal{M}\left(\mathcal{C}_{L}\right)\right|\right)$.

\section{Uncited references}

[9]

\section{Acknowledgements}

The authors are very grateful to the referees for very helpful comments.

\section{References}

[1] N. Caspard, B. Monjardet, The lattices of closure systems, closure operators, and implicational systems on a finite set: A survey, Discrete Applied Mathematics 127 (2) (2003) 241-269.

[2] V. Duquenne, The core of finite lattice, Discrete Mathematics 88 (1991) 133-147.

[3] T. Eiter, G. Gottlob, Identifying the minimal transversals of a hypergraph and related problems, SIAM Journal on Computing 24 (6) (1995) 1278-1304.

[4] T. Eiter, G. Gottlob, Hypergraph transversal computation and related problems in logic and AI, in: European Conference on Logics in Artificial Intelligence (JELIA'02), 2002, pp. 549-564.

[5] M.L. Fredman, L. Khachiyan, On the complexity of dualization of monotone disjunctive normal forms, Journal of Algorithms (21) (1996) 618-628.

[6] B. Ganter, R. Wille, Formal Concept Analysis: Mathematical Foundations, Springer-Verlag, Berlin, 1996.

[7] J.L. Guigues, V. Duquenne, Families minimales d'implications informatives resultant d'un tableau de donnes binaires, Mathétiques et Sciences humaines 95 (1986) 5-18.

[8] R. Freeze, K. Jezek, J.B. Nation, Free Lattices, American Mathematical Society, Providence, RI, 1995.

[9] P. Janssen, L. Nourine, A simplicial scheme for meet-semidistributive lattices and interval collapsing, Universalis Algebra 50 (2) (2003) 171-178.

[10] D. Maier, The Theory of Relational Data Bases, Computer Science Press, Rockville, MD, 1983.

[11] J.B. Nation, Unbounded semidistributive lattices, Algebra and Logic 39 (2000) 87-92.

[12] L. Nourine, Une structuration algorithmique de la théorie des treillis, Habilitation à diriger des recherches, Université de Montpellier II, France, July 2000. 
[13] R.C. Shock, Computing the minimum cover of functional dependencies, Information Processing Letters 3 (22) (1986) 157-159.

[14] M. Wild, Optimal implicational bases for finite modular lattices, Quaestiones Mathematicae 23 (2000) 153-161.
[15] R. Wille, Subdirect decomposition of concept lattices, Algebra Universalis 17 (1983) 275-287.

53 